\title{
Endoscopic Management of Leaks Following Laparoscopic Sleeve Gastrectomy: The Experience of IRCAD Taiwan
}

\author{
Chien-Hua Lin ${ }^{1,2 *}$, Jing-Jim Ou' ${ }^{1}$, Yueh-Tsung Lee ${ }^{1}$, Hurng-Sheng $W^{1,2}$, Sheng-Lei Yan ${ }^{3}$ \\ ${ }^{1}$ Department of Surgery, IRCAD Taiwan, Chinese Taipei \\ ${ }^{2}$ Department of Surgery, Tri-Service General Hospital, Chinese Taipei \\ ${ }^{3}$ Division of Gastroenterology, Department of Internal Medicine, Chang-Bing Show Chwan Memorial Hospital, Chinese Taipei \\ Email: *linjh93@yahoo.com.tw
}

How to cite this paper: Lin, C.-H., Ou, J.-J., Lee, Y.-T., Wu, H.-S. and Yan, S.-L. (2020) Endoscopic Management of Leaks Following Laparoscopic Sleeve Gastrectomy: The Experience of IRCAD Taiwan. Surgical Science, 11, 347-353. https://doi.org/10.4236/ss.2020.1111036

Received: October 12, 2020 Accepted: November 15, 2020 Published: November 18, 2020

Copyright $\odot 2020$ by author(s) and Scientific Research Publishing Inc. This work is licensed under the Creative Commons Attribution International License (CC BY 4.0).

http://creativecommons.org/licenses/by/4.0/

\begin{abstract}
Background: To present the experience of management of leaks following laparoscopic sleeve gastrectomy for morbid obesity in Show-Chwan memorial Hospital. Patients and Methods: Laparoscopic sleeve gastrectomy is considered one of the surgical options for morbid obesity. It is effective, with an average loss of $50 \%$ of excessive weight after 2 years of follow-up. The first laparoscopic sleeve gastrectomy was performed in January 2010 at Show-Chwan Memorial Hospital. Between January 2010 and October 2016, 300 patients underwent laparoscopic sleeve gastrectomy for morbid obesity. There were 218 women and 82 men with a mean age of 35.4 years. Preoperative mean body weight was $90.7 \mathrm{~kg}$ and mean body mass index (BMI) was $37.3 \mathrm{~kg} / \mathrm{m}^{2}$. Results: Mean operative time was 85 minutes. Mean hospital stay was 3.5 days. There were no deaths. There were 5 complications (1.67\%): leakage of gastroesophageal junction. One patient was conservative treatment. Two patients were successfully treated by self-expandable metallic stents and the other two patients were treated with esophageal stent also, but failed and further treated with fibrin (Histoacryl) injection to the leak site to try to seal the fistula. In six months follow-up, mean BMI decreased from 37.3 to 29.2 $\mathrm{kg} / \mathrm{m}^{2}$, and mean excess weight loss reached $42.8 \%$. Conclusions: Laparoscopic sleeve gastrectomy is a beneficial operation in terms of excessive weight loss, with acceptably complications. The leaks were located in gastroesophageal junction mostly, and could be resolved with esophageal stent. For the patients failed in esophageal stents, we try Histoacryl injection to improve it.
\end{abstract}

\section{Keywords}

Bariatric Surgery, Leaks, Stent, Histoacryl 


\section{Introduction}

More and more people suffer from morbid obesity in the past several decades. The most effective therapy to treat obese and related comorbidities is bariatric surgery, in which Roux-en-Y gastric bypass and sleeve gastrectomy are two most popular procedures. Sleeve gastrectomy is a surgical procedure first used as an initial step in the management of patients with severe obesity [1]. Due to simple and good results in loss of weight, it's more applied [2] [3]. However, complications need to be mentioned, such as: weight regain and gastro-esophageal reflux disease. The most important and feared complication is a gastric leak. The incident of leak was reported at an average of $2.7 \%$ from 24 studies with 1749 patients, and many approaches have been employed for the management of this complication [3]-[8]. We reported our experience of management of leaks after laparoscopic sleeve gastrectomy.

\section{Materials and Methods}

\subsection{Patients}

Consecutive 300 cases of sleeve gastrectomy were performed from January 2010 to October 2016 at IRCAD Taiwan. The surgical procedure was performed by the same surgeon, experienced in advanced laparoscopic surgery. One of the patients had undergone previous bariatric surgery of adjustable band. Among these 300 patients, 5 complications were observed. There were 218 women and 82 men with a mean age of 35.4 years. Preoperative mean body weight was $90.7 \mathrm{~kg}$ and mean body mass index (BMI) was $37.3 \mathrm{~kg} / \mathrm{m}^{2}$.

\subsection{Results}

The demographics of these patients were shown in Table 1.

There were 5 complications (1.67\%), leakage of GE junction. In our series, we will observe the vital signs of patients and the amount of leakage. One patient received conservative treatment due to the vital sign was stable and the amount of leakage was no more than $50 \mathrm{cc} /$ day. This patient recovered well and removed the drain in $21^{\text {st }}$ post-operative day.

However, if patients presented with high volume of leakage and tachycardia, fever. We will try self-expandable metallic stents (SEMSs) for the first treatment. Two patients were successfully treated by SEMSs and the other two patients were treated with SEMSs also, but failed and further treated with fibrin (Histoacryl) injection to the leak site to try to seal the fistula.

\subsection{Surgical Technique}

Self-expandable metallic stents (SEMSs) (Evolution, $12.5 \mathrm{~cm}$, Cook Endoscopy, Winston-Salem, North Carolina, USA):

Patients are positioned with supine position under general anesthesia. Upper GI series revealed a leak near the GE junction (Figure 1). An endoscopy was 
done to localize the leak then place the stent under C-arm guided. Repeat endoscopy to check the whether the stent covered the site of leaks (Figure 2), or injection of gastrografin to check. Two patients were successfully treated with SEMSs.

Table 1. Characteristics of patients with leaks following laparoscopic sleeve gastrectomy.

\begin{tabular}{ccccc}
\hline Variable & Age & Sex & Time to diagnosis (days) & Treatment \\
\hline 1 & 26 & M & 2 & SEMS + drainage \\
2 & 41 & F & 21 & SEMS + drainage \\
3 & 48 & F & 7 & Repair with drainage + SEMS + histoacryl \\
4 & 26 & F & 456 & SEMS + histoacryl + brush + drainage \\
5 & 50 & M & 2 & NPO, antibiotics \\
\hline
\end{tabular}

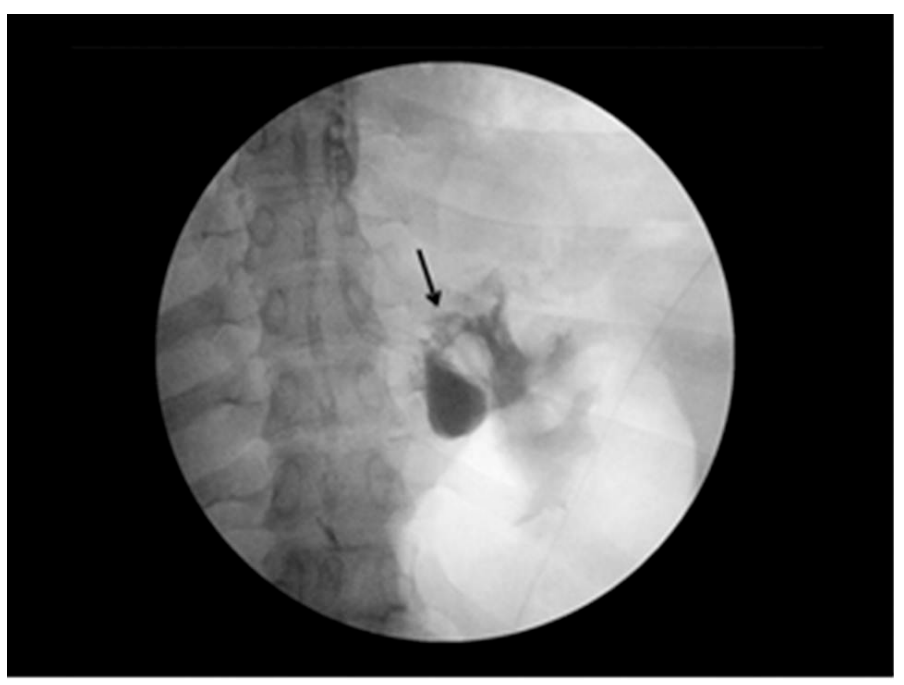

Figure 1. Upper GI series shows the extravasation of gastrograffin in GE junction. (Black arrow).

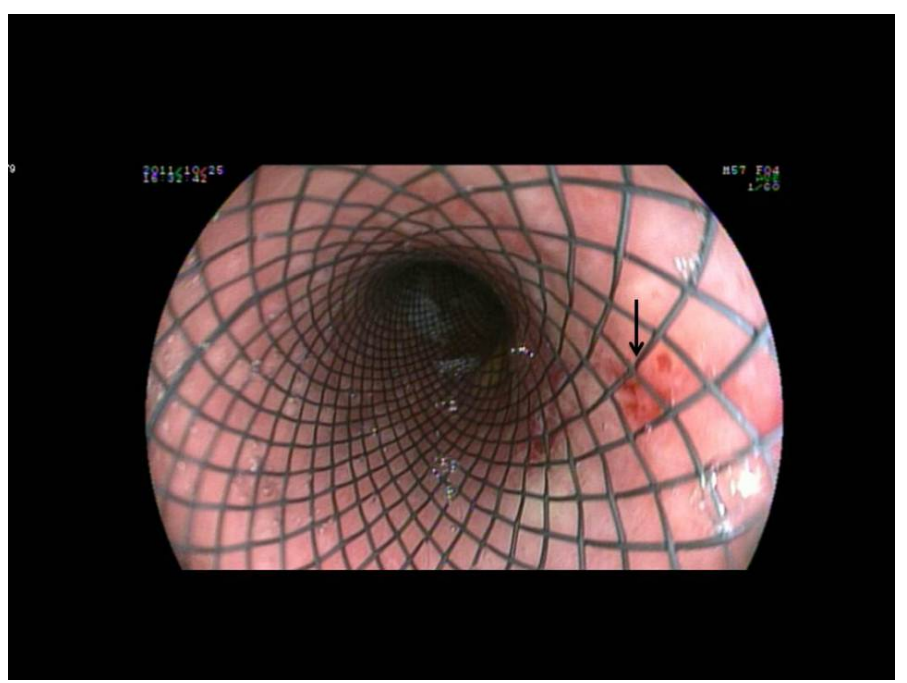

Figure 2. The endoscopy shows whether the stent covered the site of leaks. 


\section{Self-expandable metallic stents (SEMSs) plus Histoacryl injection:}

We have two patients received SEMs and histoacryl injection. A 48-year-old woman developed leaks on postoperative day 7 , then received emergent laparotomy, which revealed a staple line leak near the esophagogastric junction. Three weeks later, an esophagocutaneous fistula was seen on a barium esophagogram. We placed a partially covered SEMS (Evolution, $12.5 \mathrm{~cm}$, Cook Endoscopy, Winston-Salem, North Carolina, USA) for her. Owing to prominent tissue ingrowth at both uncovered ends of the SEMS after 5 weeks later, and difficulty for removal. We applied overtube to help to remove SEMS [9]. Several days later, a fistula developed then we injected histoacryl to seal the hole. No recurrence was found during one year follow-up.

Another patient is a 29 years old female, presented with gastric fistula in 15 months following laparoscopic sleeve gastrectomy. We used the SEMs first to treatment, but failure. Then, we try injection of histoacryl to the fistula site and also failed. Then we try endoscopic brushing (Figure 3 ) to destroy the epithelium of fistula plus injection of histoacryl. Brushing the epithelium of gastric fistula would make fistula bleeding (Figure 4). This fistula closed after three months follow-up.

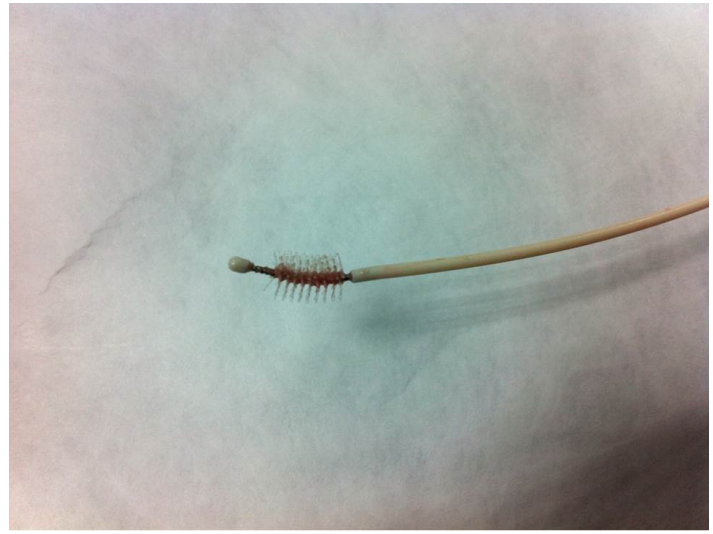

Figure 3. We used the endoscopic brush to destroy the epithelium of gastric fistula.

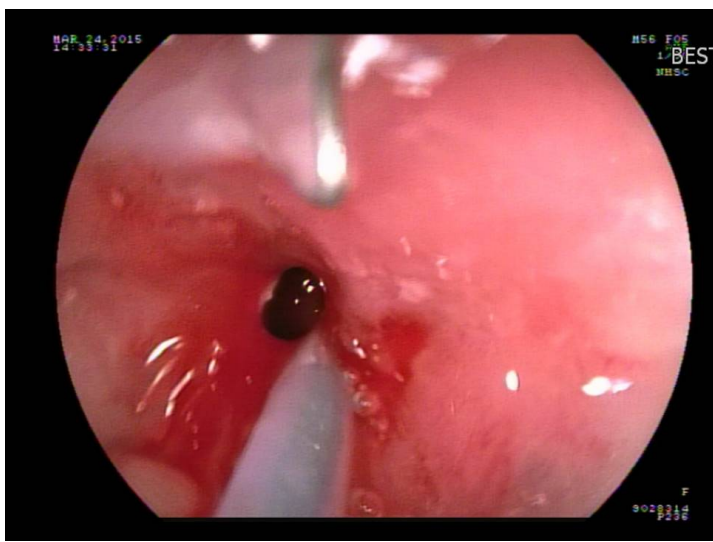

Figure 4. After destroying the epithelium of gastric fistula, the gastric fistula will bleed. Then prepare for injection of Histoacryl. 


\section{Discussion}

Sleeve gastrectomy has become a commonly performed procedure used for obesity due to its relative simple compared with other procedures such as gastric bypass, especially in Asian. However, it could make life-threatening complications, such as leaks. The occurrence rate ranges from $0.5 \%$ to $5 \%$ [3] [4] [5].

Leak management depends on the time of the diagnosis. During the first day of the postoperative period, a relaparoscopy should be performed [3]. For the late leaks, first thing is percutaneous drainage of the fluid collection. After controlling the intra-abdomnial peritonitis, the next thing is to control the leaks. All of our patients underwent implantation of SEMs. In our data, two cases were successfully, and another two patients presented persistent leaks. For the patients with persistent leaks, we further treated with injection of histoacryl.

Histoacryl ( $n$-Butyl-2-Cyanoacrylate) is a kind of tissue adhesives. Tissue adhesives can be divided into two categories: organic and inorganic. Fibrin glue, an organic product, is routinely used in surgical procedures to stop low-pressure bleeding and has been used in a variety of other applications. It is a blood product, and its mechanism of action relies on the formation of fibrin from the precursors, fibrinogen and thrombin. Histoacryl is an inorganic resin such as histoacryl are compounds that polymerize in an exothermic reaction, forming a mechanical bond between adjacent surfaces. [10] There have been case reports applied in GI fistulas endoscopically with fibrin tissue glue, lipiodol, or histoacryl [11] [12] [13]. In our cases, we have two cases underwent endoscopic injection of histoacryl, one is successful and another one is failed. However, there were some tricks, the key point of injection of Histoacryl is "quick injection and not spilled out" because Histoacryl could be rapidly polymerized in contacted with moisture (Figure 5).

The case No. 4 is a patient with chronic fistula. We tried SEMs and injection of histoacryl initially, and recurrence was found after removal of SEMs. Then we tried injection of histoacryl again. However, it's failed. Therefore, we tried applied endoscopic brushing (Figure 3 \& Figure 4) to destroy the epithelium of

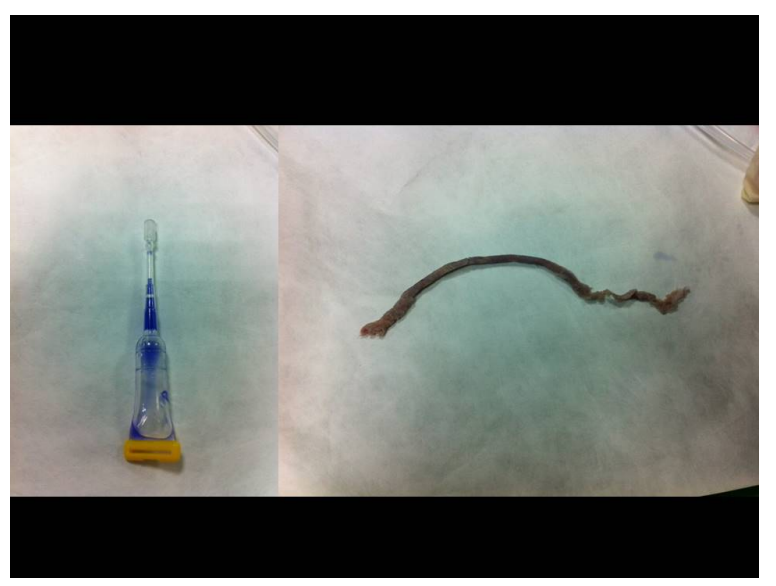

Figure 5. The key point of injection of Histoacryl is "quick injection and not spilled out" because Histoacryl could be rapidly polymerized in contacted with moisture. 
fistula then injection of histoacryl to seal the hole. No evidence of fistula was observed at oral contrast study one week and one month after therapy.

\section{Conclusion}

In conclusion, laparoscopic sleeve gastrectomy is a fetal complication and difficulty to treat. We apply SEMS and percutaneous drainage for the first line treatment. If failure, we could try histoacryl injection via gastroscopy. Endoscopic brushing plus injection of histoacry seems a good method for the treatment of chronic fistulas in patients who fail conservative medical treatment and are not operative candidates.

\section{Conflicts of Interest}

The authors declare no conflicts of interest regarding the publication of this paper.

\section{References}

[1] Regan, J.P., Inabnet, W.B., Gagner, M., et al. (2003) Early Experience with Two Stage Laparoscopic Roux Y Gastric By-Pass as an Alternative in the Upper Obese Patient. Obesity Surgery, 13, 61-64. https://doi.org/10.1381/096089203322618669

[2] Cottam, D., Quereshi, F.G., Mattar, S.G., et al. (2006) Laparoscopic Sleeve Gastrectomy as an Inital Wieght Loss Procedure for High Risk Patients with Morbid Obesity. Surgical Endoscopy, 20, 859-863. https://doi.org/10.1007/s00464-005-0134-5

[3] de Aretxabala, X., Leon, J., Wiedmaier, G., et al. (2011) Gastric Leak after Sleeve Gastrectomy: Analysis of its Management. Obesity Surgery, 21, 1232-1237. https://doi.org/10.1007/s11695-011-0382-5

[4] Clinical Issues: Committee of American Society for Metabolic and Bariatric Surgery (2010) Update Position Statement on Sleeve Gastrectomy as a Bariatric Procedure. Surgery for Obesity and Related Diseases, 6, 1-6.

[5] Serra, C., Baltasar, A., Andreo, L., et al. (2007) Treatment of Gastric Leaks with Coated Self expanding Stents after Sleeve Gastrectomy. Obesity Surgery, 17, 866-872. https://doi.org/10.1007/s11695-007-9161-8

[6] Salinas, A., Baptista, A., Santiago, E., et al. (2006) Self Expandable Metal Stents to Treat Gastric Leaks. Surgery for Obesity and Related Diseases, 2, 570-572. https://doi.org/10.1016/j.soard.2006.08.007

[7] Burgos, A.M., Braghetto, I., Csendes, A., et al. (2009) Gastric Leak after Laparoscopic Sleeve Gastrectomy. Obesity Surgery, 19, 1343-1348.

https://doi.org/10.1007/s11695-009-9884-9

[8] Nguyen, N.T., Nguyen, X.-M.T. and Dholakia, C. (2010) The Use of Endoscopic Stent in Management of Leaks after Sleeve Gastrectomy. Obesity Surgery, 20, 1289-1292. https://doi.org/10.1007/s11695-010-0186-Z

[9] Yan, S.L., Chen, C.H., Yeh, Y.H., et al. (2011) Endoscopic Removal of an Embedded Partially Covered Esophageal Self-Expandable Metallic Stent by Overtube Technique. Endoscopy, 43, E400-E401. https://doi.org/10.1055/s-0030-1256945

[10] Mahajan, A., Rao, G., Lees, G., et al. (2008) A Case of Successful Ablation of Gastrophrenia Fistula with n-Butyl-2-Cyanoacrylate. Canadian Journal of Gastroenterology, 22, 69-70. https://doi.org/10.1155/2008/516416 
[11] Lee, Y.C., Na, H.G., Suh, J.H., et al. (2001) Three Cases of Fistulae Arising from Gastrointestinal Tract Treated with Endoscopic Injection of Histoacryl. Endoscopy, 33, 184-186. https://doi.org/10.1055/s-2001-11670

[12] Ozdil, B., Yamak, Y.Z., Kece, C., et al. (2011) Successful Endoscopic Therapy of Postoperative Duodenal Fistula by Lipiodol Injection: A New Therapeutic Approach. Minimally Invasive Therapy \& Allied Technologies, 20, 193-196. https://doi.org/10.3109/13645706.2010.518791

[13] Papavramidis, T.S., Kotzampassi, K., Kotidis, E., et al. (2008) Endoscopic Fibrin Sealing of Gastrocutaneous Fistulas after Sleeve Gastrectomy and Biliopancreatic Diversion with Duodenal Switch. Journal of Gastroenterology and Hepatology, 23, 1802-1805. https://doi.org/10.1111/j.1440-1746.2008.05545.x 